\title{
Modelling the dynamic behaviour of small scale junction fires using curvature flows
}

\author{
J.J. Sharples ${ }^{\text {ab }}$, J. Hilton ${ }^{\mathrm{cb}}$ \\ ${ }^{a}$ School of Physical, Environmental and Mathematical Sciences, University of New South Wales Australia, \\ ${ }^{\mathrm{b}}$ Bushfire and Natural Hazards Cooperative Research Centre, East Melbourne, Australia \\ ${ }^{\mathrm{c}}$ CSIRO, Melbourne, Australia., \\ Email: j.sharples@adfa.edu.au
}

\begin{abstract}
The merging of two lines of fire is a relatively common occurrence in landscape fire events. For example, it can arise through the coalescence of two wildfires or when a prescribed fire meets a wildfire as part of suppression efforts. When two fires approach one another, the effects of convective and radiative heat transfer are compounded and high rates of spread can arise as a result. This is particularly the case when two lines of fire meet at some oblique angle - the point of intersection on the newly merged fire can advance rapidly. This case was investigated recently by Viegas et al. (2012), who devised an analytical model to emulate the effects of energy concentration between the two merging fire lines. In this paper, we present a more geometric approach by considering the evolution of the merged fire as the flow of a plane curve with a normal speed that depends on the curvature of the fire front. Parametric variation of the curvature dependence is investigated, and the resultant geometric evolutions of the fire front are compared with the experimental observations of Viegas et al. (2012). Further theoretical aspects of plane curvature flows and their more general application to fire front modelling are also discussed.

Junction fires occur when two fire lines meet at an oblique angle. The interaction of the two fire lines means that junction fires can exhibit unexpected fire behaviour, with enhanced rates of spread in the vicinity of the junction point. Junction fires also comprise one of the main fire behaviours involved in spot fire coalescence. Quantifying the interaction between two oblique fire lines is essential for the development of next generation fire spread models, which will allow for prediction of dynamic fire propagation. Previous work considered using fire line curvature as a simple means for modelling the dynamic behaviour of junction fires. However, in this preliminary work the method for modelling the fire spread was based on initial conditions that were not truly representative of merging fire lines. Subsequent work has implemented the curvature based model using a level set method, which allows for more natural boundary conditions. This approach was used to successfully simulate the behaviour of large-scale experimental grassland fires. In this paper we consider the same approach applied to experimental fires that are an order of magnitude smaller than those considered previously. Optimal parameters for the curvature based model applied to the smaller scale fires are derived and compared to the optimal parameters obtained for the larger scale fires. This comparison reveals information about how the dynamic behaviour of junction fires varies across different scales.
\end{abstract}

Keywords: Wildfire simulation, fire line merging, level set method, fire line curvature 


\section{INTRODUCTION}

Fire behaviour modelling is traditionally aimed at determining, to within a reasonable degree of accuracy, the rate of spread and fire behaviour characteristics of a single line of fire. This is reflected in the empiricallybased models that form the benchmark for fire behaviour modelling in Australia and in many other parts of the world (Sullivan, 2009). These models perform reasonably well when used to predict the behaviour of single fires that burn under uniform conditions similar to those upon which the models were derived, but can perform quite poorly when confronted with situations involving more sophisticated dynamics. In some such situations the assumption of quasi-steady spread inherent in empirically-based models, whereby a fire is assumed to spread at a quasi-constant rate of spread uniquely determined by a given set of environmental conditions, can be violated and a fire can behave in ways that are distinctly at odds with the traditional models.

Indeed, there are now many examples in which fires behave in a distinctly dynamic manner, in violation of the quasi-steady spread assumption. Viegas and Pita (2004), Viegas (2005) and Dold and Zinoviev (2009) describe the case of eruptive fire spread, where fires can exhibit exponential increases in rate of spread in terrain that is steep and confined, but which is otherwise unchanging, while Sharples et al. (2012) and Simpson et al. (2013) discuss the existence of dynamic modes of fire spread on steep lee-facing slopes driven by the interaction of the fire's plume with terrain-modified winds. These examples demonstrate how dynamic interactions can produce substantial increases in rate of spread despite negligible changes in environmental conditions.

Viegas et al. (2012) consider yet another example of dynamic fire spread, namely the merging of two oblique lines of fire. The interaction of the two oblique fire fronts greatly enhances the convective and radiative heat transfer processes that drive fire propagation and can result in the very rapid advance of the merged front. In these fires, which we refer to as junction fires, it was noted that the point of intersection exhibited an abrupt increase in the rate of spread followed by a gradual decrease in the rate of spread over the course of time. It was also found that the increased rate of spread became more pronounced as the angle between the two fire lines was reduced. To account for this phenomenon Viegas et al. (2012) proposed an analytic model based on the distribution of energy produced by the two fire fronts near the point of intersection. The predictions of the model were found to be in reasonable agreement with the results of laboratory scale experiments.

Sharples et al. (2013) presented an alternative approach to modelling the evolution of junction fires. In this approach the rate of spread of the merged fire line was assumed to depend on the curvature of the fire line. In essence, fire line curvature was used as a geometric surrogate, or proxy, to describe the compounding of heat near the point of intersection. Sharples et al. (2013) demonstrated that fire line curvature produced results that were in qualitative agreement with the experimental observations of Viegas et al. (2012).

However, the analytical model considered by Sharples et al. (2013) had a number of shortcomings: the merged fire line was modelled as an open curve, rather than a closed interface delineating burnt and unburnt regions; and the merged fire line was considered as being of infinite extent - this lead to the imposition of boundary conditions that prevented more quantitative comparison of the model predictions with the experimental data. In this paper these shortcomings are directly addressed by considering the modelled fire line as a closed curve, whose evolution is tracked numerically using a more appropriate numerical methodology (the level set method). Such an approach permits the inclusion of more faithful boundary conditions and hence better scope for comparison between the model behaviour and the experimental observations of Viegas et al. (2012).

It should be noted that in this study we consider the evolution of the merged fire line burning on flat ground, in homogeneous fuels and in the absence of wind. This exactly emulates the experimental conditions of Viegas et al. (2012).

\section{METHODS}

\subsection{Fire line curvature and curvature flow}

At any point in time we consider the merged fire front as being described by a closed plane curve $\gamma(t)$, with $t \in[0, \infty)$ denoting the particular point in time. This is in contrast to Sharples et al. (2013) who considered the fire front as an open curve. The evolution of the merged fire front is modelled by the normal flow of $\gamma(t)$. Formally, the evolution of the curve $\gamma(t)$ via normal flow is determined by the system of partial differential equations:

$$
\mathbf{n} \cdot \partial_{t} \gamma=s
$$


where $\mathbf{n}$ denotes the unit normal vector field to the curve $\gamma$ and $s$ denotes the normal speed; that is, the rate of advancement of any point on the curve in the direction of the normal vector field at that point.

As described above, to account for the enhanced rate of spread of the point of intersection of the merged fire lines we consider the effect of fire line curvature on the local rate of spread. Formally, fire line curvature, denoted $\kappa$, is defined as the divergence of the unit normal vector field $\mathbf{n}$ of the curve $\gamma(t)$ representing the fire line; that is,

$$
\kappa=\nabla \cdot \mathbf{n}
$$

We then assume that the normal speed $s$ is some functional of the fire line curvature: $s=s(\kappa)$. In this instance we refer to the normal flow as a curvature flow. Specifically, in this work we consider

$$
s(\kappa)=\beta-\alpha \kappa,
$$

where $\alpha$ and $\beta$ are model parameters, which are to be determined.

We note that the form of (3) implies that the normal speed will increase in the presence of negative curvature and decrease in the presence of positive curvature. We assume this form to emulate the compounding of heat energy that would be expected ahead of concave parts of the fire perimeter (i.e. where the outward pointing normal converges in on itself).

Numerical implementation of the fire line evolution model defined by equations (1) and (3) is accomplished using the level set method (Sethian, 2001; Smereka, 2003).

\subsection{The level set method}

The level set method provides a means for tracking an interface $\gamma$. In the present case of fire spread $\gamma$ is the fire front, which marks the interface between burnt and unburnt fuel. Level set methods have been applied in many areas such as geometric flow problems, image enhancement, gas-phase combustion, crystal growth and fluid mechanics (Sethian, 1999). For the case of fire spread the level set method treats the one-dimensional interface (fire front) $\gamma(t)$ as the zero level set of a higher-dimensional function $\phi: \mathbb{R}^{2} \times[0, \infty) \rightarrow \mathbb{R}$. The evolution of $\phi$ is then linked to the propagation of the front through an initial value problem, so that at any time the front is given as the zero level set of the (time-dependent) function $\phi$ (Sethian, 1999), that is:

$$
\gamma(t)=\{\mathbf{x}: \phi(\mathbf{x}, t)=0\}
$$

Since the unit normal to $\gamma$ is then given by $\mathbf{n}=\nabla \phi /|\nabla \phi|$, it follows from equation (1) that

$$
\partial_{t} \phi+s|\nabla \phi|=0
$$

For the case of curvature flow, we have

$$
\kappa=\nabla \cdot \frac{\nabla \phi}{|\nabla \phi|}=\frac{\nabla^{2} \phi}{|\nabla \phi|}-\frac{\nabla \phi}{|\nabla \phi|^{2}} \nabla \cdot(|\nabla \phi|) .
$$

Hence, given (3), equation (5) can be written as:

$$
\partial_{t} \phi-\alpha \nabla^{2} \phi+N(\phi)=0
$$

where the nonlinear term $N(\phi)$ is given by

$$
N(\phi)=\alpha \frac{\nabla \phi}{|\nabla \phi|} \cdot \nabla(|\nabla \phi|)+\beta|\nabla \phi|
$$

Equation (7) is a nonlinear parabolic equation for the distance function $\phi$, which will be considered over the triangular domain depicted in Figure 1. The domain is designed to emulate and accommodate the experimental configurations considered by Viegas et al. (2012). To fully specify $\phi$, and hence the front $\gamma(t)$, we must augment equation (7) with initial and boundary conditions that are relevant to the particular fire spread situation under consideration. 


\subsection{Initial and boundary conditions}

We take the initial state of the fire front to be the simple closed curve $\gamma_{0}$ as illustrated in red in Figure 1 . The angled components of $\gamma_{0}$ are given by:

$$
\gamma_{0}(x)=\left\{\begin{aligned}
-x \cot \left(\frac{\theta}{2}\right), & x \in[-L, 0] \\
x \cot \left(\frac{\theta}{2}\right), & x \in(0, L] .
\end{aligned}\right.
$$

In precise terms $\phi(\mathbf{x}, 0)=\gamma_{0}$ defines the initial condition for equation (7).

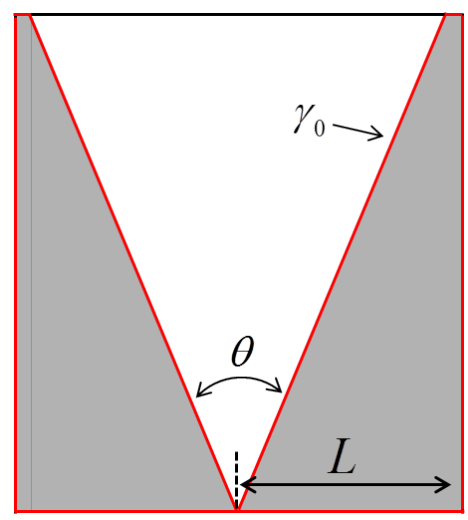

Figure 1. Model domain and the initial interface $\gamma_{0}$ (red curve), separating burnt fuel (grey) with unburnt fuel (white). In accordance with Viegas et al. (2012) we assume that the initial fire lines are $6 \mathrm{~m}$ in length, which implies that $L=6 \sin \left(\frac{\theta}{2}\right)$.

To properly specify the evolution of the interface we also need to prescribe boundary conditions at the top of the domain; that is, along the line $y=L \cot \left(\frac{\theta}{2}\right)$. The boundary conditions are prescribed so that they emulate the pattern of fire spread seen in the experiments of Viegas et al. (2012). In this work we assume the fixed boundary conditions as:

$$
\frac{\partial \phi}{\partial x}=\frac{\partial \phi}{\partial y}=0, ; \text { when } y=L \cot \left(\frac{\theta}{2}\right)
$$

The level set method is then implemented numerically using the methodology outlined by Hilton (2014).

\section{RESULTS}

The system of equations $(7,8)$ was solved subject to the boundary conditions $(10)$, for various renderings of the initial interface and for different values of the parameter values $\alpha$ and $\beta$. The simulated evolution of the fire front was compared to the fire front evolution as observed by Viegas et al. (2012).

\subsection{Evolution of the merged fronts}

Figure 2 shows how the simulated fire lines compare with observed fire for the case of $\theta=10^{\circ}$, for twelve different combinations of the model parameters $\alpha$ and $\beta$. It should be noted that no attempt was made to optimise the model parameters so that the model produced a 'best' fit. Figure 2 demonstrates that there is a relatively small range of values for $\alpha$ and $\beta$ over which the model produces a reasonable quantitative fit to the data, at least in the early stages of the evolution of the merged fire lines.

Indeed, the results indicate that the best model performance is achieved when $\alpha \approx 0.004$ and $\beta \approx 0.008$. The figure also indicates that the model performance is inconsistent, with more accurate prediction at the start of the simulation and less accurate position towards the end as the fire progression begins to slow. Having said this, however, the model does also exhibit a gradual decrease in the rate of advance of the merged fire line, just not in a way that is consistent with the observations. 


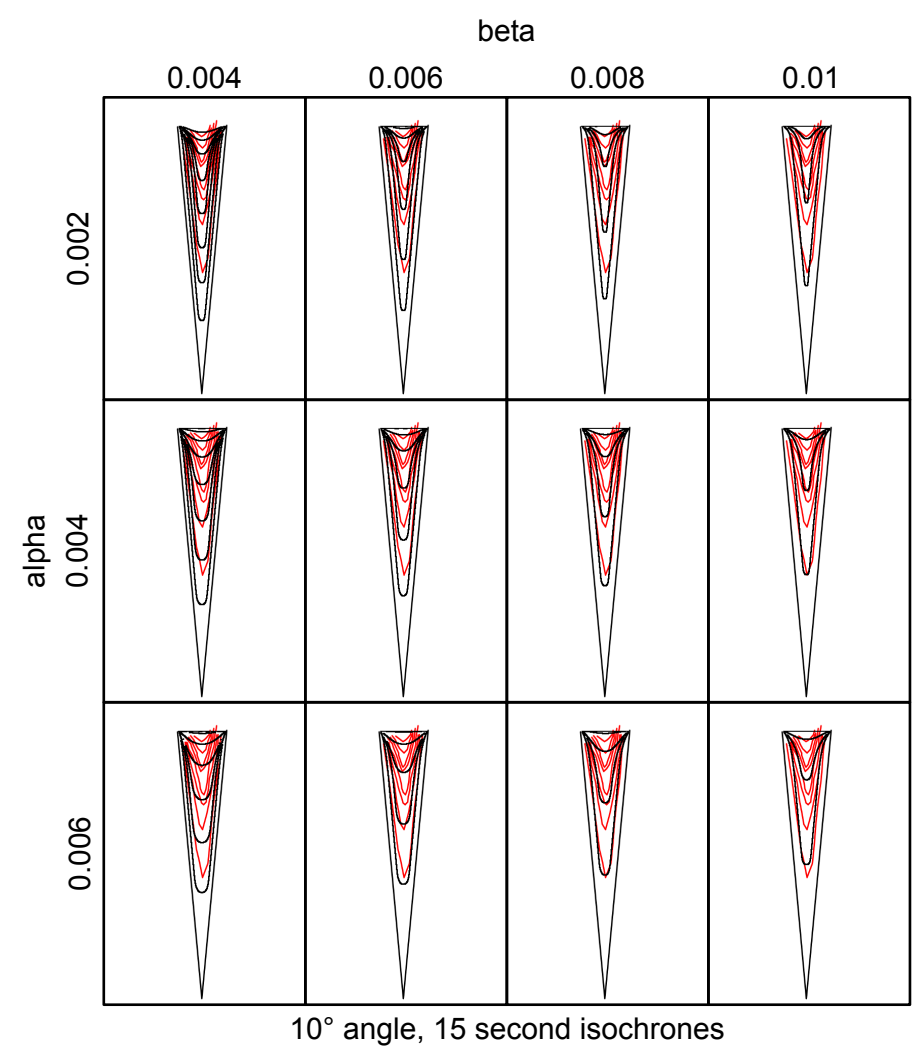

Figure 2. Simulated merged fire line evolution overlayed with the experimental observations of Viegas et al. (2012) for $\theta=10^{\circ}$. The model parameters defining the simulation output are indicated top and left.

The over-estimation of the rate of spread in the later stages of the evolution is possibly not as concerning as it may appear, as it is likely that the fire spread is being affected by the proximity of the front to the end of the experimental plot. It is likely that near the top of the experimental plot (where the fuel has been removed) the fire spread is being affected by factors that are not well-accounted for using a curvature term; for example, the entrainment of cooler air into the fire front. Away from this top boundary, however, the curvature-dependent model does a reasonable job of reproducing the observed evolution of the merged fire line.

Figure 3 shows the simulated evolution of the merged fire line for $\theta=30^{\circ}$ and compares it to the corresponding data of Viegas et al. (2012). In this case the model provides the most accurate reproduction of the observed fire behaviour when the model parameters were taken as $\alpha \approx 0.002$ and $\beta \approx 0.003$. In contrast, with the $\theta=10^{\circ}$ case, for which the model only performed well in the initial stages of the simulation, the model in this case provided more consistently accurate performnace throughout the whole simulation.

It is of interest to note that the values of $\alpha$ and $\beta$ giving the best match to observations were quite different for each of the $\theta=10^{\circ}$ and $\theta=30^{\circ}$ cases. The cases of $\theta=20^{\circ}$ and $\theta=45^{\circ}$, were also considered (not shown) and exhibited similar results. For the $\theta=20^{\circ}$ case, the best model performance was found when $\alpha \approx 2-4 \times 10^{-3}$ and $\beta \approx 4-6 \times 10^{-3}$, while for the $\theta=45^{\circ}$ case, the best model performance was found when $\alpha \approx 1-2 \times 10^{-3}$ and $\beta \approx 2-3 \times 10^{-3}$. Taken in total the results indicate that the model parameters $\alpha$ and $\beta$ decrease in an approximately logarithmic manner as the angle $\theta$ increases.

\section{DISCUSSION AND CONCLUSION}

Instances of dynamic fire spread pose a significant threat to the safety of firefighters and the community. The rapid rates of spread, like those seen in junction fires, could cause firefighters to be overrun when caught between two merging fire lines, for example. In addition, the greater fire line intensities that are associated with higher rates of spread means that junction fires, or indeed any dynamic fire mechanism that produces unusually high rates of spread, will have implications in terms of their environmental impacts. In this context it is interesting to note that current operational modelling frameworks are not able to account for the feedbacks that give rise to this type of dynamic behaviour. In this paper a curvature-based model that accounts for a 


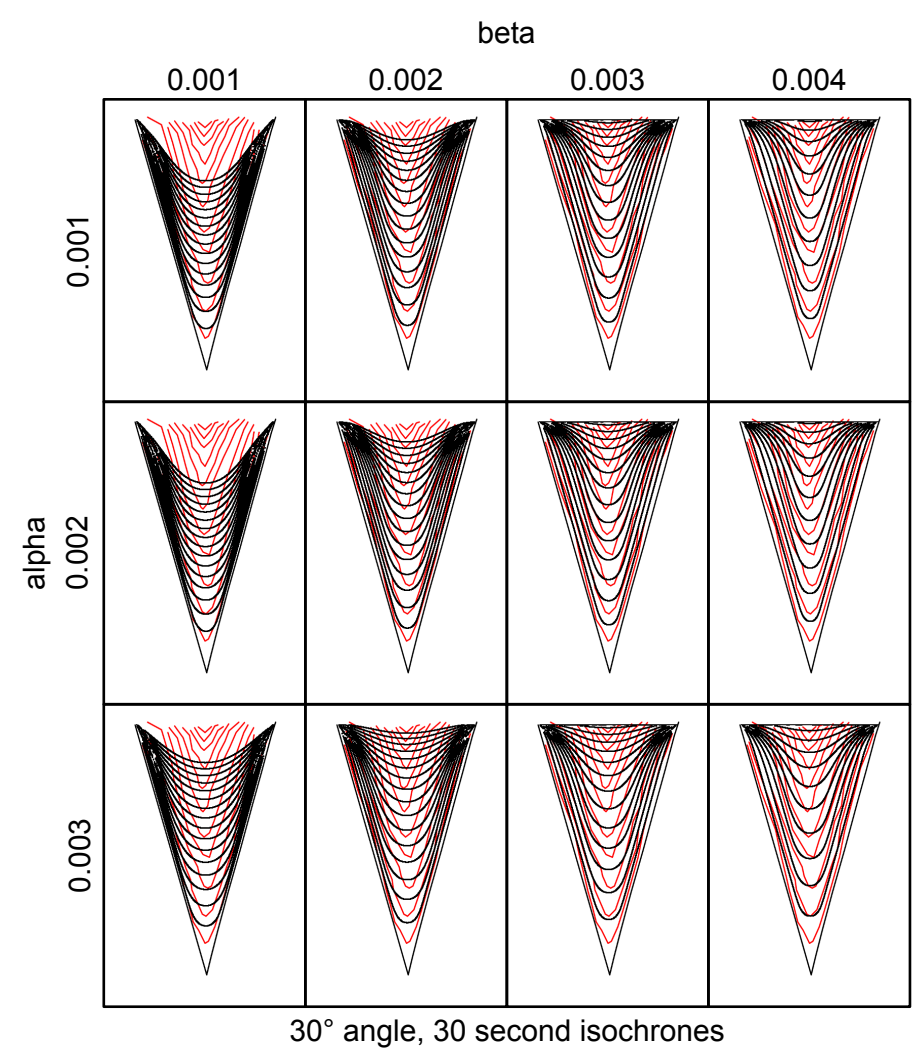

Figure 3. Simulated merged fire line evolution overlayed with the experimental observations of Viegas et al. (2012) for $\theta=30^{\circ}$. The model parameters defining the simulation output are indicated top and left.

number of aspects of dynamic fire spread associated with junction fires was investigated.

The model was conceptualised as a geometric flow with curvature-dependent speed. The properties of such flows have been well-studied (Sethian, 1985; Osher and Sethian, 1988). In particular, it has been proven (mathematically) that certain geometric flows with curvature-dependent speed have the property that any sharp corner or cusp point in the initial data will be immediately smoothed (Angenent, 1990, 1991; Altschuler and Grayson, 1992; Ecker and Huisken, 1999). Such a property is consistent with the behaviour of fire perimeters observed during experimental programs (Anderson et al., 1982). The normal flow approach was implemented using a level set method to track the evolving interface between burnt and unburnt fuel. Different values of the model parameters $\alpha$ and $\beta$ were chosen so that the model simulations provided reasonable quantitative agreement with the patterns of fire spread observed in the experiments of Viegas et al. (2012).

The level set approach allowed for a number of improvements in the modelling approach compared to that used by Sharples et al. (2013). Treating the evolving front as a closed curve rather than a graph permitted a more sensible implementation of the boundary conditions, which provided model simulations that better matched the behaviour of the experimental fires near the (no fuel) boundary at the top of the domain of interest. The fact that the fixed boundary conditions produced model results that better accounted for the pattern of spread at the top of the domain is interesting. The boundary conditions used above stipulate that there is no burning in both (normal and transverse) directions along the boundary. However, the authors are unaware of any obvious reason why a fire should not burn, in a direction that is parallel to the boundary, into unburnt fuel along the boundary. The reason must have something to do with the local pyrogenic flows near the boundary, but the particular details of these flows and how they produce conditions that are consistent with the fixed boundary conditions used above would need to be investigated with more sophisticated modelling or more refined experimentation. Such work is currently being conducted.

Another short-coming of the curvature-based approach is the fact that the model parameters producing the best predictions depended on the angle $\theta$. Indeed, both model parameters were found to decrease as $\theta$ increased. These findings are also consitent with the findings of Hilton et al. (2016), who considered curvature flow models applied to wind-driven, landscape-scale experimental fires. For such fires they found that the best 
Sharples and Hilton, Modelling the dynamic behaviour of small scale junction fires

predictions were given by $\alpha=1.05$ and $\beta=0$ for a junction fire with $\theta \approx 60^{\circ}$, and by $\alpha=1.95$ and $\beta=0$ for a junction fire with $\theta \approx 30^{\circ}$. Taken as a whole, the results suggest that not only are the curvature-based model parameters dependent on the angle $\theta$, but also on the scale of the fire being considered.

\section{REFERENCES}

Altschuler, S. J. and M. A. Grayson (1992). Shortening space curves and flow through singularities. Journal of Differential Geometry 35, 283-298.

Anderson, D., E. Catchpole, N. DeMestre, and T. Parkes (1982). Modelling the spread of grass fires. Journal of the Australian Mathematical Society, Series B 23, 451-466.

Angenent, S. (1990). Parabolic equations for curves on surfaces.part i: Curves with p-integrable curvature. Annals of Mathematics 132, 451-483.

Angenent, S. (1991). Parabolic equations for curves on surfaces.part ii: Intersections, blow-ups and generalized solutions. Annals of Mathematics 133, 171-215.

Dold, J. W. and A. Zinoviev (2009). Fire eruption through intensity and spread rate interaction mediated by flow attachment. Combustion Theory and Modelling 13, 763-793.

Ecker, K. and G. Huisken (1999). Mean curvature evolution of entire graphs. Annals of Mathematics 130, $453-471$.

Hilton, J. (2014). Level set methods for fire fronts. Technical Report EP141393, CSIRO Computational Informatics, Clayton, Victoria, Australia.

Hilton, J., C. Miller, J. Sharples, and A. Sullivan (2016). Curvature effects in the dynamic propagation of wildfires. International Journal of Wildland Fire 25(12), 1238-1251.

Osher, S. and J. A. Sethian (1988). Fronts propagating with curvature-dependent speed: algorithms based on Hamilton-Jacobi formulations. J. Comp. Phys. 79(1), 12-49.

Sethian, J. (1999). Level set methods and fast marching methods (2nd ed.). Cambridge University Press.

Sethian, J. (2001). Evolution, implementation and application of level set and fast marching methods for advancing fronts. Journal of Computational Physics 169, 503-555.

Sethian, J. A. (1985). Curvature and the evolution of fronts. Communications in Mathematical Physics 101, 487-499.

Sharples, J., R. McRae, and S. Wilkes (2012). Wind-terrain effects on the propagation of large wildfires in rugged terrain: fire channelling. International Journal of Wildland Fire 21, 282-296.

Sharples, J., I. Towers, G. Wheeler, V.-M. Wheeler, and J. McCoy (2013). Modelling fire line merging using plane curvature flow. In J. Piantadosi, R. Anderssen, and J. Boland (Eds.), MODSIM2013, 20th International Congress on Modelling and Simulation, pp. 256-262. Modelling and Simulation Society of Australia and New Zealand.

Simpson, C., J. Sharples, J. Evans, and M. McCabe (2013). Large eddy simulation of atypical wildland fire spread on leeward slopes. International Journal of Wildland Fire 22, 282-296.

Smereka, P. (2003). Semi-implicit level set method for curvature and surface diffusion motion. Journal of Scientific Computing 19, 439-456.

Sullivan, A. L. (2009). Wildland surface fire spread modelling, 1990-2007. 2. Empirical and quasi-empirical models. International Journal of Wildland Fire 18, 369-386.

Viegas, D. (2005). A mathematical model for forest fires blow-up. Combustion Science and Technology 177, 27-51.

Viegas, D. and L. Pita (2004). Fire spread in canyons. International Journal of Wildland Fire 13, $253-274$.

Viegas, D., J. Raposo, D. Davim, and C. Rossa (2012). Study of the jump fire produced by the interaction of two oblique fire fronts. Part 1: Analytical model and validation with no-slope laboratory experiments. International Journal of Wildland Fire 21, 843-856. 